\title{
Variable rotational line broadening in the Be star Achernar ${ }^{\star}, \star \star$
}

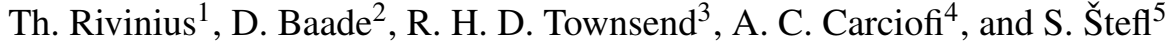 \\ ${ }^{1}$ ESO - European Organisation for Astron. Research in the Southern Hemisphere, Casilla 19001, Santiago, Chile \\ e-mail: triviniu@eso.org \\ 2 ESO - European Organisation for Astron. Research in the Southern Hemisphere, Karl-Schwarzschild-Str. 2, Garching, Germany \\ 3 Department of Astronomy, Univ. of Wisconsin-Madison, 2535 Sterling Hall, 475 N. Charter Street, Madison, WI 53706, USA \\ ${ }^{4}$ Instituto de Astronomia, Geofísica e Ciências Atmosféricas, Universidade de São Paulo, 05508-900 São Paulo SP, Brazil \\ 5 ESO/ALMA - The Atacama Large Millimeter/Submillimeter Array, Alonso de Córdova 3107, Vitacura, Santiago, Chile \\ Received 20 August 2013 / Accepted 26 September 2013
}

\section{ABSTRACT}

\begin{abstract}
Aims. The main theoretical problem for the formation of a Keplerian disk around Be stars is how angular momentum is supplied from the star to the disk, even more so since Be stars probably rotate somewhat subcritically. For instance, nonradial pulsation may transport angular momentum to the stellar surface until (part of) this excess supports the disk-formation/replenishment. The nearby Be star Achernar is presently building a new disk and offers an excellent opportunity to observe this process from relatively close-up. Methods. Spectra from various sources and epochs are scrutinized to identify the salient stellar parameters characterizing the disk life cycle as defined by $\mathrm{H} \alpha$ emission. The variable strength of the non-radial pulsation is confirmed, but does not affect the other results. Results. For the first time it is demonstrated that the photospheric line width does vary in a Be star, by as much as $\Delta v \sin i \lesssim 35 \mathrm{~km} \mathrm{~s}^{-1}$. However, unlike assumptions in which a photospheric spin-up accumulates during the diskless phase and then is released into the disk as it is fed, the apparent photospheric spin-up is positively correlated with the appearance of $\mathrm{H} \alpha$ line emission. The photospheric line widths and circumstellar emission increase together, and the apparent stellar rotation declines to the value at quiescence after the $\mathrm{H} \alpha$ line emission becomes undetectable.
\end{abstract}

Key words. line: profiles - stars: rotation - stars: emission-line, Be - stars: individual: $\alpha$ Eri

\section{Introduction}

Be stars are rapidly rotating stars that form gaseous, circumstellar viscous decretion disks (see Rivinius et al. 2013a, for a review). To form a viscous decretion disk and keep it in existence, angular momentum must be supplied to the inner part of the disk, which is then transported outwards by viscosity (see, e.g., Haubois et al. 2012). It is generally considered that Be stars rotate close to, but not at a critical value, so that there has to be a disk formation process acting on top of the rotation. Since the disk is transient in many Be stars, either the formation or the dissipation process of the disk, or both, must be able to vary with time in strength for a given star. Among the proposed mechanisms for this is the pulsational build-up of excess angular momentum in the upper photosphere that eventually "offloads" into a circumstellar disk (Ando 1986; Rogers et al. 2013; Neiner et al. 2013). Such a mechanism should leave an imprint on the photosphere by increasing the rotational line widths, usually expressed as $v \sin i$, before the disk is formed/supplied with angular momentum, and then should decrease as the disk forms. To our knowledge, no observational results have been published from searching for such a phenomenon.

The southern Be star Achernar ( $\alpha$ Eri, HD 10144) is the Be star with the brightest apparent magnitude. It is mostly

* Based on observations collected at the European Southern Observatory at La Silla and Paranal, Chile, Prog. IDs: 62.H-0319, 64.H0548, 072.C-0513, 073.C-0784, 074.C-0012, 073.D-0547, 076.C-0431, 077.D-0390, 077.D-0605, and the technical program IDs 60.A-9120 and 60.A-9036.

$\star \star$ Appendices are available in electronic form at http://www . aanda.org classified as B3 V or B4 IV, but sometimes also B6 V. Vinicius et al. (2006) attempted to reconcile these discrepant spectral types and other published parameter values by taking the rapid rotation of the star into account, and they derived a "surface mean effective temperature" of $15000 \mathrm{~K}$. Achernar's rapid rotation is typical of Be stars, but it is the only Be star for which the rotational flattening has actually been observed (Domiciano de Souza et al. 2003). Domiciano de Souza et al. (2012) revisited the star and, based on more interferometric data, confirmed the initial findings of Achernar rotating at about $95 \%$ of the critical velocity. However, the determinations of the actual projected rotational speed differ quite widely: Vinicius et al. (2006) obtained $v \sin i=223 \pm 15 \mathrm{~km} \mathrm{~s}^{-1}$, while Domiciano de Souza et al. (2012) give $292 \pm 10 \mathrm{~km} \mathrm{~s}^{-1}$.

Achernar is also known to be a non-radial pulsator, with a pulsation frequency of $F_{1}=0.775 \mathrm{c} / \mathrm{d}$, which is seen in both spectroscopy and photometry (Rivinius et al. 2003, and references therein). A somewhat more detailed picture was obtained by Goss et al. (2011) based on satellite photometry. They found the above-mentioned period to be stable in value and phase, but in amplitude correlated with the circumstellar activity state; i.e., when circumstellar emission is present, the amplitude is high. A stronger, secondary frequency $F_{2}$, at a value about $10 \%$ lower, was found to only be present at times of circumstellar activity, which is often seen in Be stars (Štefl et al. 1998).

\section{Observations}

High-quality echelle spectra are required for the analysis. The data sets available for this study (see Table 1) were obtained 


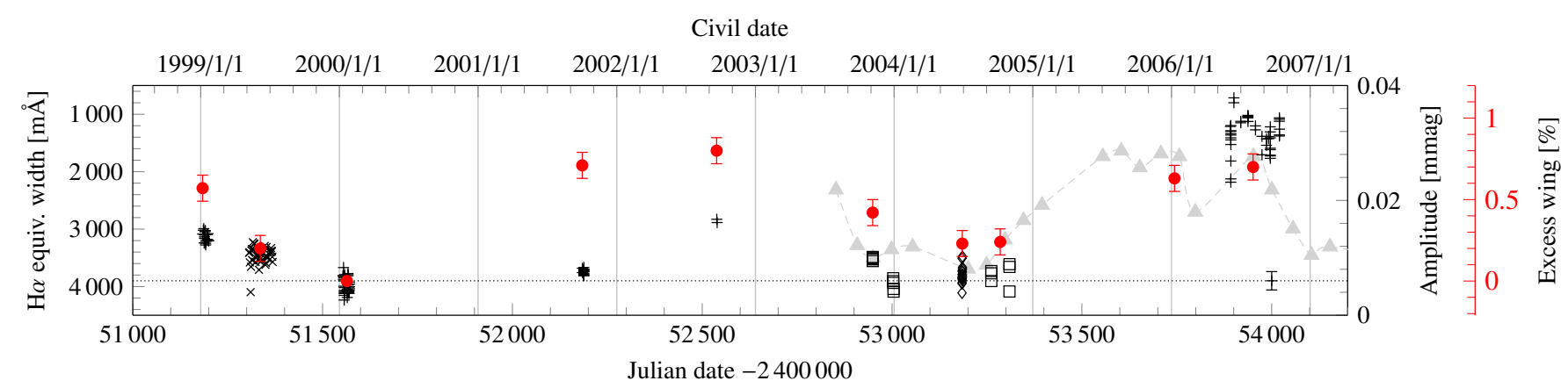

Fig. 1. Achernar H $\alpha$ equivalent width 1999 to 2007. + for FEROS, $\times$ for HEROS, $\square$ for HARPS, and $\diamond$ for UVES. The mean value in diskless state, $3900 \mathrm{~m} \AA$, is indicated as a dotted line. The mean uncertainty of $\pm 160 \mathrm{~m} \AA$, estimated as RMS in diskless state, is shown exemplarily in the lower right corner. Light gray triangles $(\Delta)$ are photometric amplitudes of frequency $F_{1}$ taken from Fig. 4 of Goss et al. (2011). Solid disks $(\bullet)$ mark the depth of the excess wing signature in He 4471 (average of the red and blue sides, see also Fig. 3) in the mean difference spectra.

Table 1. Observations used in this work.

\begin{tabular}{lcccc}
\hline \hline Date range & Instrument & $\begin{array}{c}\text { Coverage } \\
{[\mathrm{nm}]}\end{array}$ & $\begin{array}{c}\text { Resolv. } \\
\text { power }\end{array}$ & $\begin{array}{c}\text { \# of } \\
\text { spectra }\end{array}$ \\
\hline Jan. 1999 & FEROS & $370-890$ & 48000 & 20 \\
Jun./Jul. 1999 & HEROS & $350-860$ & 20000 & 43 \\
Jan. 2000 & FEROS & $370-890$ & 48000 & 44 \\
Oct./Nov. 2001 & FEROS & $370-890$ & 48000 & 16 \\
Sep. 2002 & FEROS & $370-890$ & 48000 & 3 \\
Sep.-Dec. 2003 & HARPS & $380-690$ & 115000 & 11 \\
Jun. 2004 & UVES & $320-950$ & 60000 & 17 \\
Sep./Oct. 2004 & HARPS & $380-690$ & 115000 & 6 \\
Jan. 2006 & UVES & $380-500$ & 60000 & 60 \\
Jun.-Dec. 2006 & FEROS & $370-890$ & 48000 & 45 \\
\hline
\end{tabular}

with the instruments HEROS (Stahl et al. 1995), FEROS (Kaufer et al. 1999), UVES (Dekker et al. 2000), and HARPS (Mayor et al. 2003). Spectra taken after the year 2000 are available from the ESO Science Archive Facility. HEROS technical parameters, observing procedures, and data reduction are described by Stahl et al. (1995). The UVES and FEROS data were reduced with the standard data reduction systems provided by ESO, and for HARPS the reduced data were obtained from the archive. Some spectra were discarded for quality reasons or because of unsuitable observing modes, e.g., HARPS spectra taken with an iodine cell. See Appendix A for a discussion of stability over several seasons and instruments. The $\mathrm{H} \alpha$ equivalent widths were then measured in each spectrum (Fig. 1). For the analysis of highquality differences between the seasons, the spectra in each run listed in Table 1 were averaged. Unity was added to the difference spectra, so that the continuum has a value of one instead of zero.

\section{Variations relative to the diskless state}

\subsection{Circumstellar contributions}

Strong emission lines have never been observed in Achernar (H $\alpha$ peak height always $\lesssim 1.5 F_{\text {cont }}$, see Vinicius et al. 2006), but even these vanished completely between July and October 1999. Spectra taken with FEROS in January 1999 and May-July 1999 with HEROS show weak emission in $\mathrm{H} \alpha$ alone, but this had disappeared fully when the star was observed in January 2000 with FEROS again. The independent FEROS observations by Vinicius et al. (2006) were taken in October 1999 and confirm the absence of any detectable disk. Taking the spectra of January 2000 as photospheric reference, one can construct the differences to test circumstellar contribution or photospheric change against this reference. By the end of 2001, a weak disk had started forming (Although in EW, Fig. 1, the new emission is balanced by simultaneous shell absorption, as clearly seen in the difference spectrum, see Appendix B.) It peaked in 2002, was almost gone again by the end of 2003, and had completely vanished by mid 2004. Thereafter there are no spectra until 2006, when a fully developed disk was present. It is interesting to compare this evolution to the report by Goss et al. (2011), where the photometric amplitudes of the two observed frequencies follow the same pattern (see Fig. 1).

The spectra taken in 2006, towards the end of a disk formation phase, when the disk had reached maximum emission, show some properties normally only seen in shell stars. The inclination values given by modeling $\left(\sim 78^{\circ}\right.$ by Domiciano de Souza et al. $2012 ; \sim 65^{\circ}$ by Carciofi et al. 2007) are rather high, but not equatorial, suggesting that Achernar might be a transition case between clear shell and clear non-shell star. Indicators of a shell-star nature for Achernar are 1) central quasi-emission components (CQE, Rivinius et al. 1999), easily seen in Ca II 3933 (see Fig. 2) and $\mathrm{Mg}_{\text {II }} 4481$; and 2) differences from the photospheric spectrum of $1999 / 2000$ below zero in the center of the lines most easily affected by circumstellar material. The circumstellar nature of these signatures is corroborated by comparison with the modeled differences below, which, not counting the Balmer emission, do not reproduce the cores of these, and only these, lines well.

\subsection{Photospheric variations}

The largest circumstellar variations are present in 2006. Apart from clear double-peaked emission and shell-absorption contributions in the usual lines (Balmer, lowly ionized metals), however, there is a third type of difference, which is not restricted to lines typically formed in the circumstellar environment, but is seen in purely photospheric lines, too, like the $\mathrm{He}_{\mathrm{I}}$ lines in the blue part of the spectrum (see Fig. 2 for He I 4388). In the UVES 2006 data, that line shows a shallower core, but excess wings, not just deeper, but reaching out a bit farther than in 2000. In other words, the photospheric line is broader in 2006 than in 2000. The excess wing signature is also seen in Ca II 3933 in Fig. 2, at a higher velocity than the circumstellar shell absorption.

The pattern visible in Fig. 2 is not only seen in all He I and the CaII lines, but also in $\mathrm{Mg}_{\text {II }} 4481$ (see Fig. 3) and Si II 4128/32 doublet (see Fig. 4), as well as in $\mathrm{C}_{\text {II }} 4267$, Fe II 5169 , Si II $6347 / 71$, or, in short, in basically all lines detected in the spectrum with a depth more than a few percent, with the notable exception of Si III 4553. The changes in the Balmer 
Th. Rivinius et al.: Variable rotational line broadening in the Be star Achernar

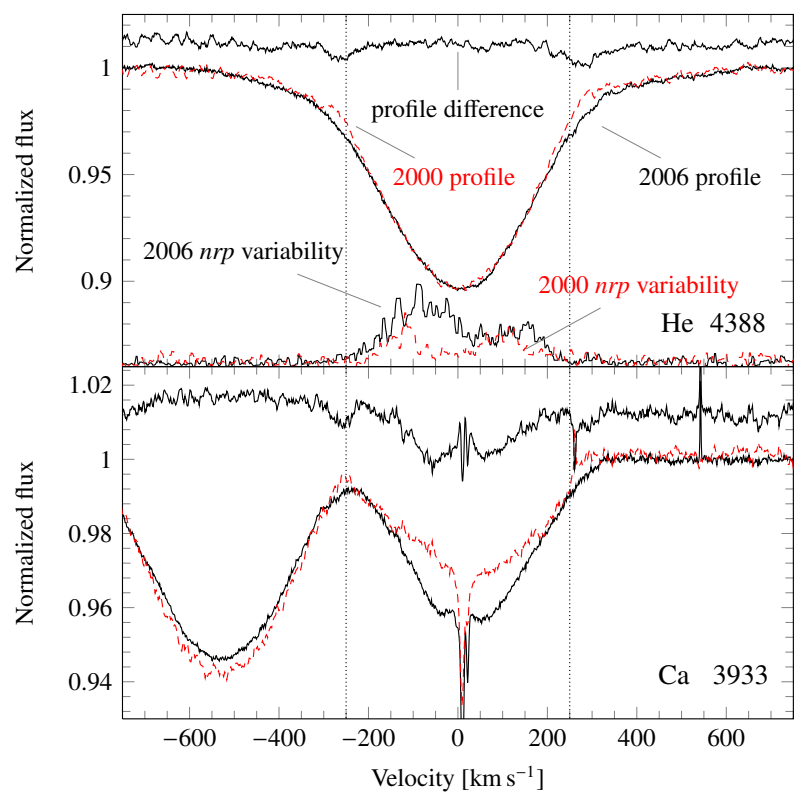

Fig. 2. FEROS 2000 (dashed, red in online version) and UVES 2006 (solid) spectra and their differences. Shown are a purely photospheric line (He I 4388) and a line with circumstellar contribution (Ca II 3933). The temporal variance spectra for the 2000 and 2006 seasons are shown for He I 4388 as well (arbitrary scaling). In neither season did the pulsational power reach beyond $\pm 250 \mathrm{~km} \mathrm{~s}^{-1}$ (dotted lines). The CQE in Ca II 3933 is embedded in a shell absorption (plus the interstellar line), but the additional absorption at high velocity is photospheric in both lines.

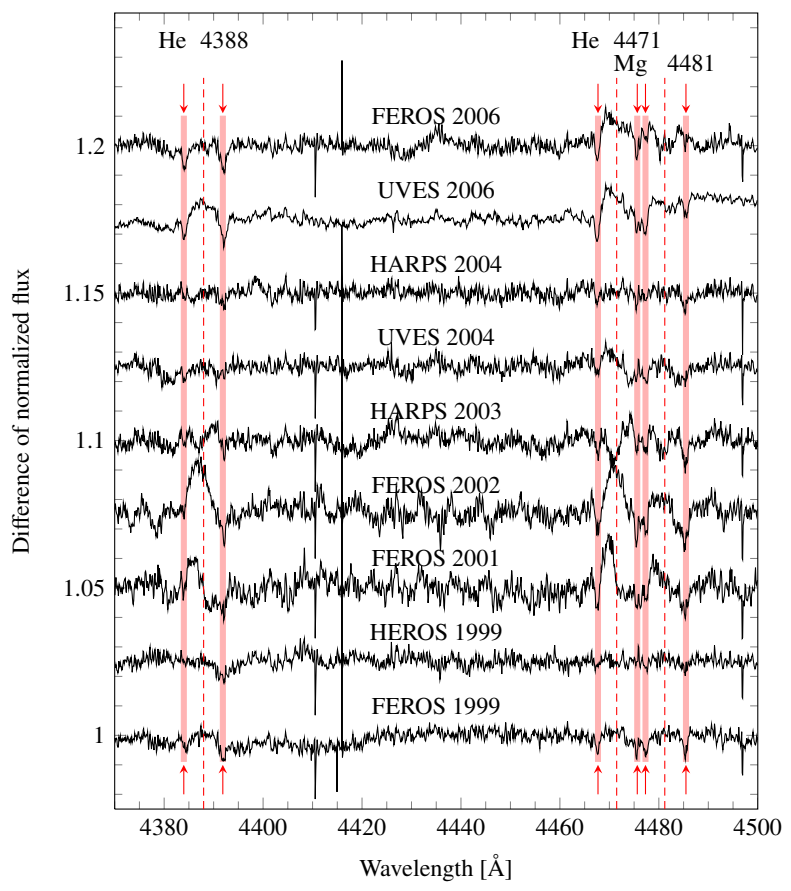

Fig. 3. Temporal evolution of the differences with respect to the pure photosphere state of 2000 (see also Fig. 1). Data were averaged for each observing epoch. In seasons with only a few observing epochs (FEROS 2001, 2002, and HARPS 2003) residual pulsational profile distortions are clearly seen in the line cores. $\mathrm{Mg}$ II 4481 shows circumstellar absorption in HARPS 2003, UVES 2006, and FEROS 2006, similar to the CQE seen in Fig. 2. Rest wavelengths of spectral lines are indicated with a dashed line, excess wing signature depression with arrows.

wings seen in Fig. 4 are probably real as well; they are consistently present in all Balmer lines except $\mathrm{H} \alpha$.
This excess broadening of all photospheric lines changes on a time scale of months to years. The temporal evolution is shown in Fig. 3, where in all epochs the excess outer wings (see Fig. 2) are deeper than in the diskless state observed with FEROS in 2000. The lines are narrowest when the star has no circumstellar disk. Indeed, the differences are quite small, and there is almost no excess broadening in the HEROS 1999 data and very little in the 2004 data, all with little to no sign of a disk. The excess broadening is strongest, i.e., clearly seen deeper and wider line wings, indicated in the differences of the spectra by "dents" at about $v \pm 275 \mathrm{~km} \mathrm{~s}^{-1}$ of each line, when the disk strength is increasing or is steady $(2001 / 2,2006)$. When the disk strength is decreasing, the excess broadening weakens (1999 and 2003). However, as 2004 data show, the excess broadening can still be weakly present when no disk emission or shell absorption can be detected anymore.

\subsection{Proof-of-concept modeling}

Since the variation is present in almost all photospheric lines in the same manner ( $\mathrm{Si}$ III 4553 can be explained by its polar formation locus), a change in the surface velocity field from epoch to epoch is a strong possibility. The variable pulsation amplitude, known from photometry, is one possibility. However, an inspection of the individual spectra does not support this hypothesis. The pulsational variations shown in an RMS variation analysis are contained well inside $\pm 250 \mathrm{~km} \mathrm{~s}^{-1}$ for the 2006 season and even within $\pm 220 \mathrm{~km} \mathrm{~s}^{-1}$ for the 2000 season. Since that means there is no variability outside that velocity, this cannot cause excess wings observed between 250 and $300 \mathrm{~km} \mathrm{~s}^{-1}$ (see Fig. 2). Pairwise differences between individual high-quality spectra also do not show any indication of the excess broadening varying as part of the clearly seen pulsational phase differences.

This variation in the excess broadening might instead come from the photospheric rotational velocity field itself being different. As a proof of concept for this hypothesis, a set of photospheric model spectra was calculated with Bruce3 (see Rivinius et al. 2013b, for a description and references). Model parameters were taken from published sources: $R_{\mathrm{pole}}=8 R_{\odot}, i=78^{\circ}, T_{\mathrm{eff}}=$ $15000 \mathrm{~K}$ from Domiciano de Souza et al. (2012), while the mass was somewhat increased from their value of $M=6.1 M_{\odot}$ to $M=8.1 M_{\odot}$ to avoid the problem of interpolating equatorial local atmosphere parameters outside the grid of model spectra available for Bruce3. It should be noted that the models used are strictly models for the photospheric appearance alone, so that a higher mass does not affect luminosity, etc., as it would in a model including the stellar interior. Only the local gravity, the geometric distortion, and the amount of gravity darkening are changed. Standard gravity darkening with $\beta=0.25$ was adopted (see Appendix $\mathrm{C}$ for a discussion of lower $\beta$ ). Spectra were computed by incrementing $v \sin i$ in steps of $5 \mathrm{~km} \mathrm{~s}^{-1}$, from 250 to $300 \mathrm{~km} \mathrm{~s}^{-1}$.

Figure 4 shows the model spectrum for $v \sin i=250 \mathrm{~km} \mathrm{~s}^{-1}$. On the scale of the figure, models computed with higher $v \sin i$ look identical. The difference spectra, however, exhibit pronounced features, which are surprisingly close to the observed ones, given that no sort of fitting beyond selecting an appropriate $\Delta v \sin i$ was attempted. The strongest discrepancies between modeled and observed difference spectra are the circumstellar absorption/emission effects identified above, which are not included in the model. Equatorially formed lines, such as singly ionized metals, are affected differently than helium lines formed over the whole star. In the equatorially formed lines, the excess wings are very strong, but the core remains largely unaltered, 


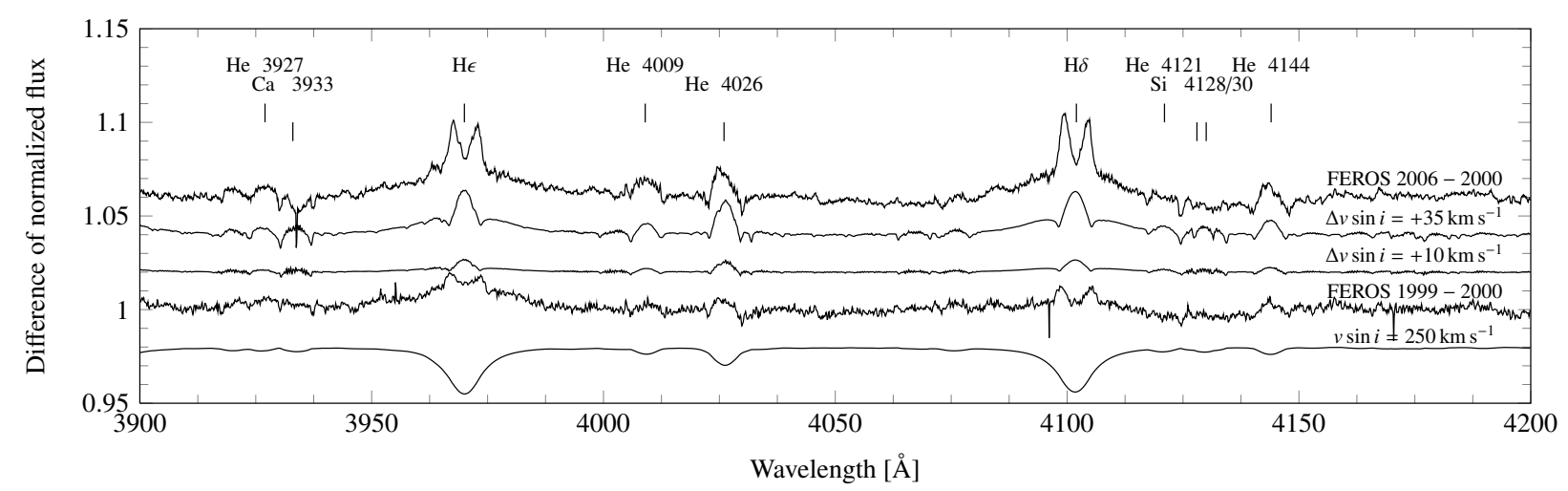

Fig. 4. As a proof of concept for a variable $v \sin i$ parameter, the differences in the FEROS 1999 and the FEROS 2006 to the FEROS 2000 spectrum are shown. Below is the modeled spectrum (scaled by a factor of 1/15), calculated with a fixed $T_{\text {eff }}=15000 \mathrm{~K}, \beta=0.25, v \sin i=250 \mathrm{~km} \mathrm{~s}^{-1}$. The model differences of $\Delta v \sin i=+35 \mathrm{~km} \mathrm{~s}^{-1}$ and $\Delta v \sin i=+10 \mathrm{~km} \mathrm{~s}^{-1}$ are shown between the observed ones.

while both excess wings and line core change for He I lines. The more polar Si III 4553 line does not change at a detectable level at all. The Balmer line wings, finally, are less deep in the rapidly rotating model owing to the lower equatorial gravity. All this can be understood in terms of the formation regions of the respective lines, as illustrated in Fig. 6 of Rivinius et al. (2013a). The effects of varying $v \sin i$ are the strongest at equatorial latitudes. This also explains why, contrary to classical assumptions, changes in $v \sin i$ do affect the equivalent width.

The observed time-dependent line broadening can be explained well by variations in the (equatorial) rotational velocity.

\section{Discussion and conclusions}

The high-quality spectroscopy of Achernar has shown a variable width for the photospheric lines. The width correlates with the disk emission. The variation can be interpreted as a change in the stellar rotation speed, and a proof-of-concept model with $\Delta v \sin i \leq 35 \mathrm{~km} \mathrm{~s}^{-1}$ already matches the observations reasonably well without parameter fitting. On top of $95 \%$ of critical rotation, such a velocity differential could make quite a difference for disk formation. However, the absence of a detectable spinup before the onset of disk formation, marked by a photometric amplitude increase in Fig. 1, puts constraints on models that first transport angular momentum upwards into the outer photosphere and then "offload" this angular momentum excess into a circumstellar disk. Instead, the excess width correlates with the presence and decay of the disk.

In a viscous disk scenario, "decay of the disk" actually means that disk material is being reaccreted onto the central star. Even in build-up and steady-state feeding phases, the very nature of viscous angular momentum transport requires that some fraction of the material in the inner disk is reaccreted. A temporary accretional spin-up of the equatorial surface region alone is another hypothesis. The enhanced velocity is dissipated back into deeper stellar regions after the reaccretion ceases, and the angular momentum required for disk formation may be taken from such deeper seated regions instead of the photospheric surface.

In any case, current models for stellar photospheres do not handle such a situation well. For instance, instead of keeping $T_{\text {eff }}$ constant with the spin-up, one could just as well keep either the polar temperature or the luminosity constant, or else implement an equatorially enhanced differential rotation and correspondingly modified local gravity/temperature values instead of a solid body spin-up; or change the values of gravity darkening and/or equatorial temperature. However, to explore and lift these possible degeneracies is far beyond the scope of this discovery report.

Since about January 2013, Achernar has been building up a new disk from almost zero emission (Napoleão \& Marcon, priv. comm., 2013). With Achernar being the closest and apparently brightest Be star, this offers a timely opportunity to obtain dedicated observations and to scrutinize whether the spin-up is cause or consequence of disk formation. Similar findings for other Be stars might be waiting for discovery in archival data.

Acknowledgements. R.H.D.T. acknowledges support from NASA award NNX12AC72G. A.C.C. acknowledges support from CNPq (307076/2012-1) and Fapesp (2010/19029-0). The referee has greatly helped improve the robustness against potential objections.

\section{References}

Ando, H. 1986, A\&A, 163, 97

Carciofi, A. C., Magalhães, A. M., Leister, N. V., Bjorkman, J. E., \& Levenhagen, R. S. 2007, ApJ, 671, L49

Dekker, H., D’Odorico, S., Kaufer, A., Delabre, B., \& Kotzlowski, H. 2000, in SPIE Conf. Ser. 4008, eds. M. Iye, \& A. F. Moorwood, 534

Domiciano de Souza, A., Kervella, P., Jankov, S., et al. 2003, A\&A, 407, L47

Domiciano de Souza, A., Hadjara, M., Vakili, F., et al. 2012, A\&A, 545, A130

Goss, K. J. F., Karoff, C., Chaplin, W. J., Elsworth, Y., \& Stevens, I. R. 2011, MNRAS, 411, 162

Haubois, X., Carciofi, A. C., Rivinius, T., Okazaki, A. T., \& Bjorkman, J. E. 2012, ApJ, 756, 156

Kaufer, A., Stahl, O., Tubbesing, S., et al. 1999, The Messenger, 95, 8

Mayor, M., Pepe, F., Queloz, D., et al. 2003, The Messenger, 114, 20

Neiner, C., Mathis, S., Saio, H., \& Lee, U. 2013, in Progress in Physics of the Sun and Stars: A New Era in Helio- and Asteroseismology, eds. H. Shibahashi, \& A. E. Lynas-Gray, ASPC, in press

Rivinius, T., Štefl, S., \& Baade, D. 1999, A\&A, 348, 831

Rivinius, T., Baade, D., \& Štefl, S. 2003, A\&A, 411, 229

Rivinius, T., Carciofi, A. C., \& Martayan, C. 2013a, A\&ARv, 21, 69

Rivinius, T., Townsend, R. H. D., Kochukhov, O., et al. 2013b, MNRAS, 429, 177

Rogers, T. M., Lin, D. N. C., McElwaine, J. N., \& Lau, H. H. B. 2013, ApJ, 772, 21

Stahl, O., Kaufer, A., Wolf, B., et al. 1995, J. Astron. Data, 1, 3

Štefl, S., Baade, D., Rivinius, T., et al. 1998, in A Half Century of Stellar Pulsation Interpretation, eds. P. A. Bradley, \& J. A. Guzik, ASPC, 135, 348

Vinicius, M. M. F., Zorec, J., Leister, N. V., \& Levenhagen, R. S. 2006, A\&A, 446,643 


\section{Appendix A: Cross-season and -instrument data}

Using data from four different instruments over about a decade to detect subtle effects might be problematic in terms of stability and cross-instrumental effects. All are echelle spectrographs; HARPS, FEROS, and HEROS are attached by fiber-link, while UVES is mounted on a gravity invariant Nasmyth platform. The three ESO instruments are monitored and partly thermally controlled for stability. All spectra were used in the heliocentric reference frame. The pixel sampling of the spectra is from $0.1 \AA$ (HEROS) over $0.03 \AA$ (FEROS) down to about $0.01 \AA$ (UVES and HARPS). This is much smaller than the width of the observed variations, which are several tens of $\mathrm{km} \mathrm{s}^{-1}$ even for the most narrow ones, the high-velocity "dents". The observed line depths differ by almost $2 \%$ of the continuum, at a typical S/N of the average spectra of about 1000 . The observed effect is not systematically different between FEROS-FEROS and OTHER-FEROS comparisons.

Since the variations are very oversampled and have a consistent appearance and evolution through all data sets, regardless of the instrument, a cross-instrumental/stability effect can be excluded.

\section{Appendix B: The $\mathrm{H} \alpha$ difference spectra}

To allow an assessment of the circumstellar disk strength independent of the $\mathrm{H} \alpha$ equivalent width, the seasonal $\mathrm{H} \alpha$ profiles are shown in Fig. B. 1 in a similar format to the one given by Vinicius et al. (2006) in their Fig. 12. In a shell star, the net effect of a weakly developed disk on the overall $\mathrm{H} \alpha$ equivalent width can vanish if, as happened in Achernar in 2001, the contributions from line emission and shell absorption just cancel one another out. Note as well the unusual Balmer-line CQE seen in the FEROS 2001 and somewhat more strongly in UVES 2004 data.

\section{Appendix C: The gravity darkening parameter}

In the proof-of-concept model, a gravity-darkening value of $\beta=0.25$ was used, even though lower values of $\beta$ are typically derived observationally. We note that $\beta=0.2$ was adopted by Domiciano de Souza et al. (2012), though not derived. The model we use can compute spectra for different values of $\beta$, but the current formalism for computing the corresponding stellar parameters, in particular $T_{\text {eff }}$ and $L$, has been derived with a fixed $\beta=0.25$. For this reason, modeling with a fixed $T_{\text {eff }}$ is only possible with $\beta=0.25$, a shortcoming that will be overcome in a more detailed dedicated work. To demonstrate that the effect does not vanish for a different $\beta$, we show models computed with constant $T_{\text {pole }}$ instead, for $\beta=0.20$ (Fig. C.1). Compared to the models shown in Fig. 4, the "dents" are unaltered in Ca II3933 and the $\mathrm{Si}$ II lines, and slightly less pronounced in $\mathrm{He}_{\mathrm{I}}$ lines, but still clearly present in He r 3927, 4009, 4121, 4144. The most significant difference is that the Balmer line wings are no longer reproduced (and Balmer lines are generally stronger), which, however, is an effect dominated by keeping $T_{\text {pole }}$ fixed rather than $T_{\text {eff }}$, and otherwise unrelated to the value of $\beta$.
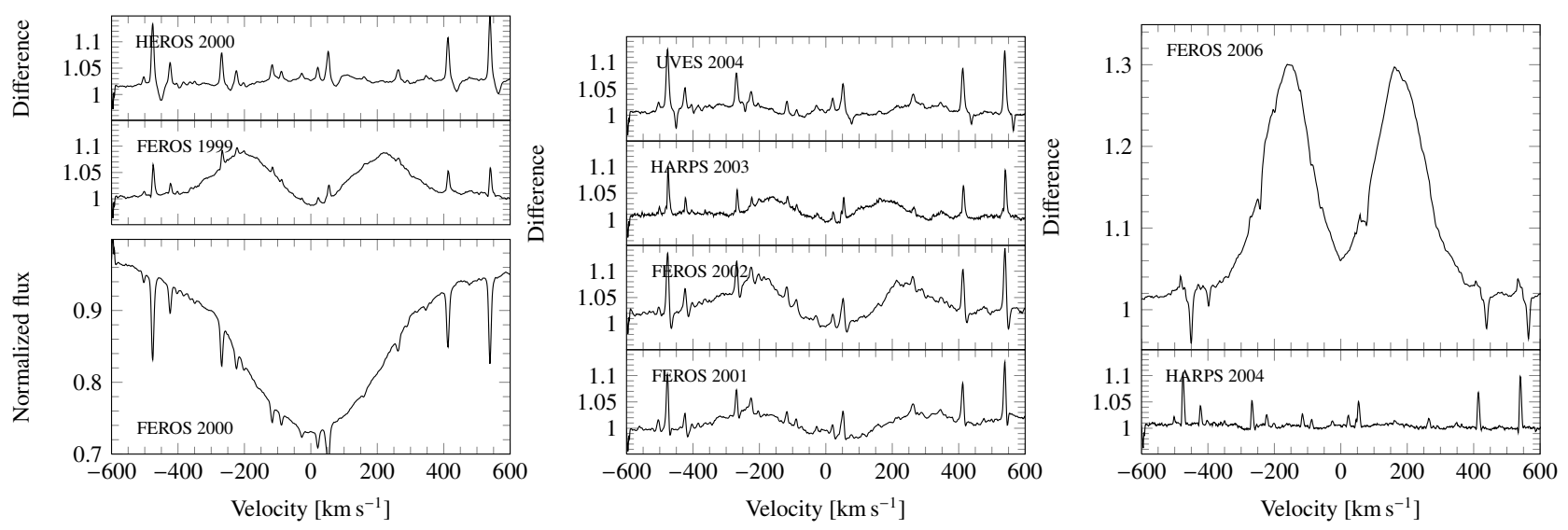

Fig. B.1. H $\alpha$ difference profiles with respect to FEROS 2000 for all observing seasons. Telluric lines were not corrected for.

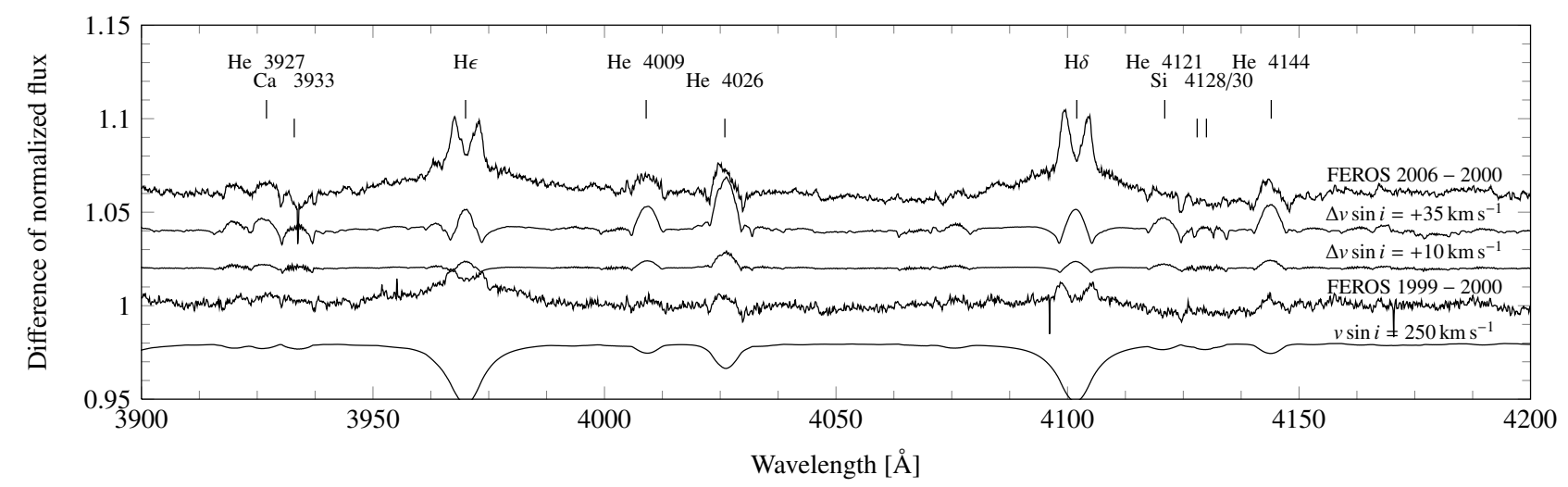

Fig. C.1. Same as Fig. 4, with models computed for a fixed $T_{\text {pole }}=16900 \mathrm{~K}$ and $\beta=0.20$. 